
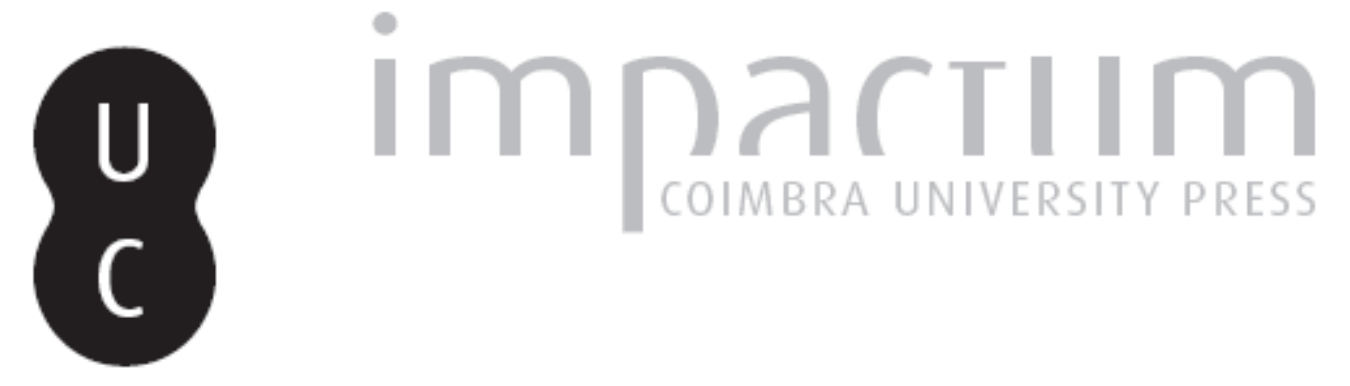

\title{
Qu'est-ce que l'ironie socratique?
}

\author{
Autor(es): Narcy, Michel
}

Publicado por: Imprensa da Universidade de Coimbra

URL persistente:

URI:http://hdl.handle.net/10316.2/42279

DOI:

DOI:https://doi.org/10.14195/2183-4105_1_2

Accessed : $\quad$ 26-Apr-2023 11:49:03

A navegação consulta e descarregamento dos títulos inseridos nas Bibliotecas Digitais UC Digitalis, UC Pombalina e UC Impactum, pressupõem a aceitação plena e sem reservas dos Termos e Condições de Uso destas Bibliotecas Digitais, disponíveis em https://digitalis.uc.pt/pt-pt/termos.

Conforme exposto nos referidos Termos e Condições de Uso, o descarregamento de títulos de acesso restrito requer uma licença válida de autorização devendo o utilizador aceder ao(s) documento(s) a partir de um endereço de IP da instituição detentora da supramencionada licença.

Ao utilizador é apenas permitido o descarregamento para uso pessoal, pelo que o emprego do(s) título(s) descarregado(s) para outro fim, designadamente comercial, carece de autorização do respetivo autor ou editor da obra.

Na medida em que todas as obras da UC Digitalis se encontram protegidas pelo Código do Direito de Autor e Direitos Conexos e demais legislação aplicável, toda a cópia, parcial ou total, deste documento, nos casos em que é legalmente admitida, deverá conter ou fazer-se acompanhar por este aviso.

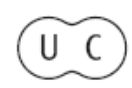




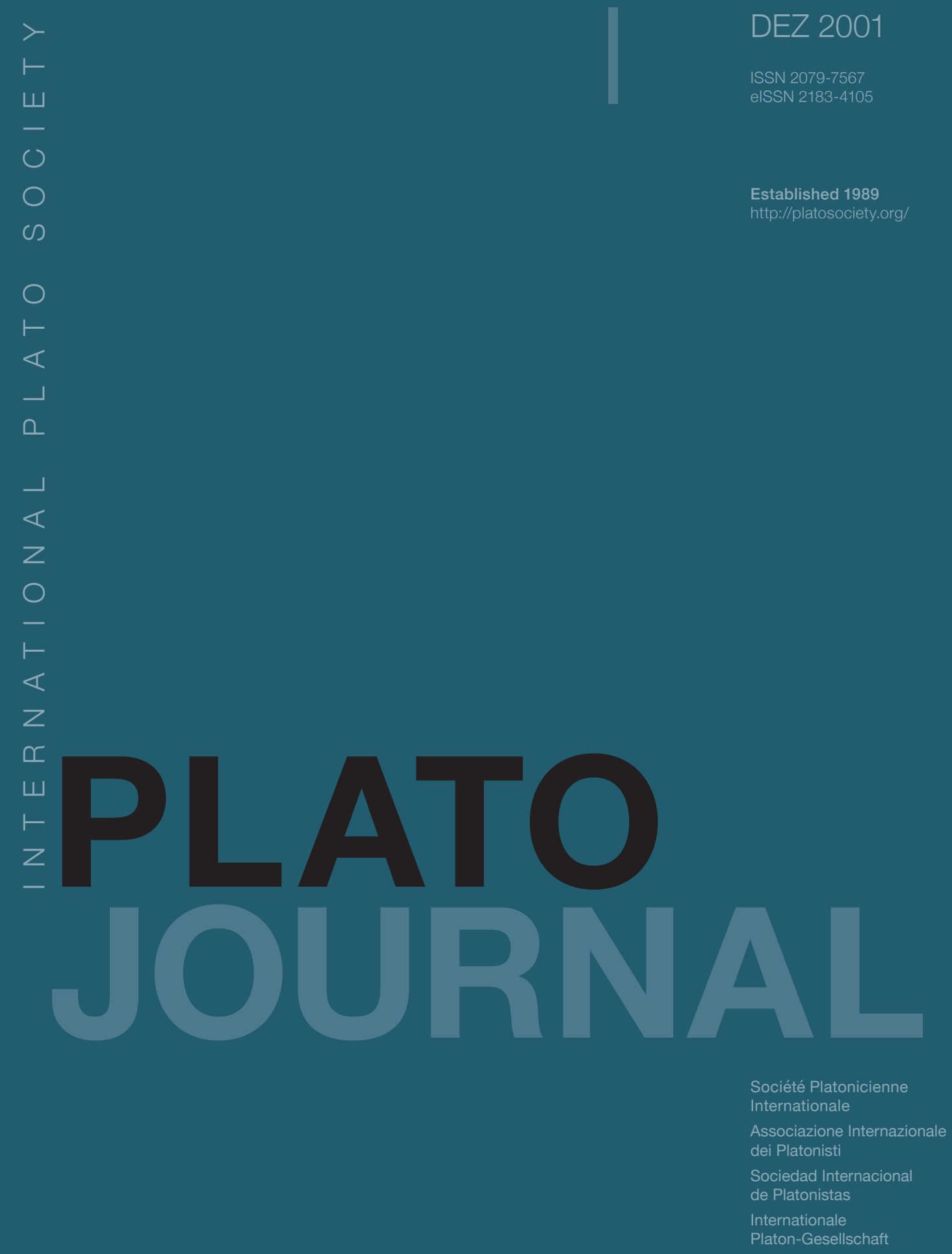




\title{
Qu'est-ce que l'ironie socratique?
}

NARCY, Michel, in 1. Plato 1 (2001), [En

ligne] , January 2001

\begin{abstract}
Socratic irony' is often understood in modern scholarship, for instance by Gregory Vlastos, as having a rather favourable sense. It is understood as implying something different from what one says (a sense later defined by Quintilian) and doing so part of a programme of moral education that consisted in challenging the intellectual powers of his interlocutors. However, this sense of eironeia is not found in Plato or in Aristotle in the texts where he discusses Socratic irony. The term 'irony' occurs in those texts in a consistently negative sense, associated with contemptuous or arrogant treatment of others (for instance in the Republic or Symposium). In Aristotle's Nicomachean Ethics, 'Socratic irony' may appear to have a more favourable sense; but close attention to the relevant passages, taken in the context of Aristotle's ethical framework, shows that it signifies a kind of wilful disdain of convention, and is no more favourably understood than the other extreme of alazoneia. If we go back to the Platonic uses of the term, for instance, Thrasymachus in Republic 337a or Callicles in Gorgias 489e, we find again a negative connotation. Here, the relevant sense is that of evading one's share in the discussion by pretending to have no answer to the questions one poses. In fact, 'evasion' is, probably, what eironeia, means, understood as a deliberate tactic to get the better of one's opponents. The more favourable sense of 'Socratic irony' is, thus, a modern invention, partly based on the more positive usage of the term in Latin, in Cicero and Quintilian.
\end{abstract}

10

C'est devenu parmi les modernes une tradition que d'associer l'ironie au personnage de Socrate, à un point tel qu'on en est venu, avec Gregory Vlastos, à en faire sa qualité principale, et à la rendre consubstantielle à sa pratique de la philosophie: au projet même de Socrate, d'exercer sur ses contemporains une sorte de magistère moral, il appartiendrait de ne le faire que de façon ironique. Voyant dans la définition donnée par Quintilien de l'ironie («faire entendre le contraire de ce qui est dit») celle qui s'applique le mieux à Socrate, Vlastos voit dans la profession socratique de non-savoir le procédé-type d'un véritable éducateur moral: questionnant et refusant de répondre, il force ses disciples à prendre eux-mêmes soin d'eux-mêmes. Moraliste dans la mesure même où il est ironiste, Socrate apparaît ainsi comme l'idéal même de l'éducateur moderne; on croirait le gouverneur de l'Émile de Rousseau: «s'il (scil. l'élève) vous fait quelque question, parlez-lui d'autre chose». L'interprétation de Vlastos est donc bien en ce sens la quintessence de l'interprétation moderne de l'ironie socratique. Vlastos le sait d'ailleurs si bien qu'allant au devant de la critique, il pose que c'est précisément avec Socrate que l'ironie passe de son acception première, dans la Grèce classique, à son acception moderne dont les premiers linéaments apparaissent chez Cicéron et Quintilien [1].

Quel était le sens d'eirôneia avant Socrate, on est d'autant plus autorisé à se le demander que la plus ancienne occurrence du mot eirôn n'est pas antérieure aux Nuéesd'Aristophane, où il désigne, sinon Socrate lui-même, du moins ce qu'on devient à son école. Comment assurer que Socrate aurait inauguré le changement d'acception d'un mot que nous ne voyons apparaitre qu'à son propos? Je voudrais montrer ici que si, incontestablement, entre l'ironie socratique et l'ironie moderne, le sens du mot «ironie» a changé, ce n'est pas à partir de Socrate, avant lequel le mot semble inconnu, mais entre Socrate et Quintilien. À l'ironie toute moderne prêtée par Vlastos à Socrate, je voudrais pour cela opposer plusieurs passages de Platon et d'Aristote, dont l'interprétation conduit à conclure à un sens d'eirôneia dont l'ironia de Cicéron et Quintilien ne semble avoir gardé aucune trace.

Alors que l'ironie attribuée à Socrate est, dans l'interprétation moderne, liée (quand elle n'en est pas purement et simplement constitutive) à sa supériorité sur ses interlocuteurs (disciples ou adversaires), elle est au contraire, dans les plus anciens témoignages qui nous en sont parvenus, systématiquement porteuse d'une connotation péjorative. Rien ne nous oblige naturellement à être d'accord avec les Anciens dans leur jugement sur Socrate, ni à donner la préférence aux traditions anti-socratiques sur celles qui lui sont favorables. Reste cependant à expliquer que la figure d'un Socrate ironiste appartienne au courant anti-socratique. Mais avant de l'expliquer, le point demande à être établi. C'est donc ce que je voudrais faire dans un premier temps, avant d'en chercher l'explication dans une définition de l'ironie qui la fait consister, non dans un trait de caractère ou une attitude psychologique, voire psychagogique, mais dans une certaine façon, dont les Anciens nous disent qu'elle fut propre à Socrate, de pratiquer la dialectique.

Que la désignation de Socrate comme ironiste soit toujours péjorative, ce n'est pas chez Platon qu'il est le plus difficile de l'établir. Lorsqu'elle est le fait de Thrasymaque (Rep. I, 337a) ou de Calliclès (Gorg. 489d), il est évident qu'il s'agit d'un reproche. Ce qui est peut-être plus difficile à admettre, c'est qu'il en va de même quand c'est Alcibiade qui comprend comme ironique le comportement de Socrate à son propre égard (Symp. 216e, 218d). Si, se dérobant à l'obligation de prononcer un éloge de l'Amour, Alcibiade annonce, dans le Banquet, un éloge de Socrate, à cet éloge se mêle bientôt l'étalage des griefs: à l'éloge, reconnaît en conclusion Alcibiade lui-même, «j'ai mêlé ce que je lui reproche en vous disant les insolences qu'il m'a faites» (ha me hubrisen) (Symp. 222a). Or ces insolences, cette hubris, c'est précisément ce qu'Alcibiade a décrit comme de l'eirôneia. Il en est question deux fois dans son 
discours: une première fois (216e4), apparaît le participe eirôneuomenos quand Alcibiade vient d'expliquer que Socrate ne se soucie ni de la beauté ni de la richesse ni d'aucune autre de ces valeurs qui, pour la foule, font le bonheur - que Socrate pense, en d'autres termes, que tout ce que l'on tient ordinairement pour des biens ne vaut rien, et que, surtout, «nous-mêmes ne sommes rien» (216d8e4): si, par ce dédain des honneurs et des richesses, Socrate ne faisait qu'illustrer l'autarcie du sage, l'ironie dont il est taxé pourrait valoir pour un éloge; mais, dès lors que, à la fin de la période, son dédain s'étend jusqu'à ses semblables, il est difficile de ne pas y voir un reproche, la première personne du pluriel employée par Alcibiade étant une façon d'impliquer son auditoire dans la réprobation qu'il fait peser sur Socrate.

La deuxième mention par Alcibiade de l'ironie de Socrate (Symp. 218d) concerne la fin de non-recevoir opposée par ce dernier à ses sollicitations érotiques, sa manière de se dérober à ses avances, alors même que, selon Alcibiade, c'est lui-même (Socrate) qui les avait provoquées. Avoir obtenu, et qui plus est de la part du bel Alcibiade, un tel retournement de la relation normale, où c'est au plus âgé de faire sa cour au plus jeune; puis, l'ayant obtenu, se refuser comme l'a fait Socrate, Alcibiade appelle cela de l'arrogance (ergon huperêphanon 217e5; ... tês Sôkratous huperêphanias 219c6): c'est que, si à nos yeux de moralistes sa résistance aux charmes d'Alcibiade est tout à son honneur, pour Alcibiade qui les lui a ouvertement offerts, elle est une humiliation, et une raison d'en vouloir à Socrate.

Nulle trace de modestie, donc, aux yeux d'Alcibiade, dans l'ironie de Socrate. Dans le passage de l'Éthique à Nicomaque où Aristote traite de l'eirôn, et où il est nommément question de Socrate (IV 7), on croit pourtant lire le contraire, puisque l'eirôn y est opposé au vantard (alazôn). Je voudrais donc examiner maintenant ce passage, pour montrer que le terme eirôn est en réalité porteur d'une connotation non moins péjorative que le mot alazôn, ce qui mène à classer ce témoignage d'Aristote parmi ceux qui sont hostiles ou du moins sévères à l'égard de Socrate, et à y voir peut-être l'origine de la tradition anti-socratique qui se développa au sein du Péripatos, illustrée notamment par Aristoxène de Tarente.

Aussi bien que la vantardise, tout d'abord, on notera que l'ironie, dans le passage aristotélicien, relève de la tromperie, en ce qu'elle s'oppose à la véracité: il en résulte que, si le véridique qui tient le milieu entre eux est digne d'éloge (1127a30-1: ho men alêtheutikos mesos ôn epainetos), le vantard et l'eirôn, son contraire (le modeste?), en tant qu'ils trompent leur monde, sont tous les deux blâmables (1127a31: hoi de pseudomenoi amphoteroi men psektoi). Mais surtout le vantard, ajoute cependant Aristote (1127a312: mallon d'ho alazôn). Et de fait, après avoir traité successivement de celui qui est enclin à la vérité (ho philalêthês) puis du vantard, quand il en vient à l'eirônet cette fois justement à Socrate, Aristote ouvre et conclut son développement par la réitération de ce déséquilibre entre les deux extrêmes. Je cite le passage dans la traduction du P. Deman, qui borne là (1127b22-26) le témoignage d'Aristote sur Socrate eirôn :

De leur côté, les «ironiques» se tiennent en deçà de la vérité (hoi de eirônes epi to elatton legontes). Ils montrent de ce chef un caractère plus gracieux: car ce n'est pas en vue d'un gain, semble-t-il, qu'ils parlent ainsi, mais pour éviter l'ostentation. Et ce sont surtout les qualités honorables que ces personnes nient posséder (malista de kai houtoi ta endoxa aparnountai): ainsi faisait Socrate.

Interprétation et délimitation du fragment ont naturellement partie liée. Ce qu'on lit dans ces lignes, c'est l'indulgence dont fait preuve Aristote à l'égard des «ironistes», indulgence qui paraît d'autant plus justifiée qu'ironie semble ici se confondre avec modestie, et que le type en est illustré justement par Socrate; mais indulgence aussi qui contraste avec les épithètes péjoratives qui servent aussitôt après à qualifier ce que l'on tient pour un autre type d' «ironistes», ceux qui, au lieu, comme Socrate, de «nier qu'ils possèdent les qualités honorables», nient posséder «les choses petites et évidentes» (ta mikra kai phanera, 1127b26).

Cette opposition de deux types d' «ironistes», dont l'un, représenté par Socrate, serait au fond digne d'éloge, et l'autre répréhensible, dépend, en réalité, d'une traduction de ta endoxa aparnountai qui, si elle fait, semble-t-il, l'unanimité, est cependant difficilement soutenable, et d'une correction qui fait elle aussi l'unanimité, mais à l'égard de laquelle j'élèverai pourtant quelques doutes.

Commençons par le point de traduction. Traduire ta endoxa aparnountai par «nier posséder les qualités honorables» ou quelque équivalent, c'est donner à chacun des éléments de ce groupe verbal une valeur probablement sans autre exemple dans le corpus aristotélicien. Tout le monde sait que, dans l'Organon, ta endoxa signifie, non pas «les qualités honorables», mais «les idées probables», «plausibles» ou, selon la traduction de J. Brunschwig, «les idées admises». Quant à aparneisthai, Bonitz n’en connaît aucun emploi en dehors des ouvres logiques d'Aristote, où il signifie: «refuser son assentiment», «refuser une prémisse». En d'autres termes, le groupe ta endoxa aparnountai peut se traduire sans difficulté dans le lexique ordinaire d'Aristote: les «ironistes» sont ceux surtout (malista) qui refusent leur assentiment aux idées admises. Que soit évoqué ici l'exemple de Socrate n'a rien de surprenant: plus qu'il ne livre un témoignage sur Socrate, Aristote souscrit à celui de Platon, dont de nombreux passages suffiraient à illustrer ce trait. Qu'on pense seulement au début du Ménon, où Socrate déclare ignorer ce qu'est la vertu, à l'Hippias majeur où il déclare ignorer ce qu'est le beau, ou au Gorgias où il se refuse à admettre qu'Archélaos, d'affranchi devenu tyran, soit heureux: autant d'idées admises, voire, pour parler comme Polos, de faits (pragmata, Gorg. 470 d 1) reconnus de tous, auxquels Socrate refuse son assentiment. Si l'on laisse aux mots, par conséquent, le sens qu'ils ont normalement chez Aristote, ce que celui-ci décrit comme de l'ironie, ce n'est pas la modestie de Socrate, mais son comportement dialectique.

Passons maintenant à la correction. Aux lignes 1127 b 26-27, la leçon des manuscrits est la suivante :

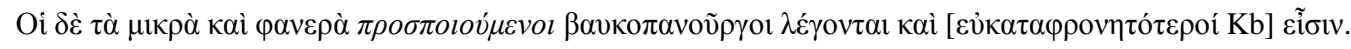


I. Vahlen (Philologus, 21 [1864], p. 153-4) a proposé de supprimer prospoioumenoi et de sous-entendre à la place aparnoumenoi, propositions adoptées depuis par l'ensemble, respectivement, des éditeurs et des traducteurs. Les arguments de Vahlen étaient les suivants: (1) prospoieisthai est affecté, dans ce chapitre, à la description de l'alazôn. Mais c'est oublier que, la première fois qu'il en parle dans l'Éthique à Nicomaque (II 7, 1108a20-2), Aristote oppose vantardise (alazoneia) et ironie (eirôneia) comme deux formes de prospoiêsis, l'une vers le plus (epi to meizon), l'autre vers le moins (epi to elatton). Rien d'insolite par conséquent si dans notre passage, ayant précisément dit des eirônes qu'ils parlent epi to elatton, Aristote utilise à leur propos le verbe prospoieisthai. (2) Les deux phrases malista de ... aparnountai et hoi de ...prospoioumenoi sont étroitement liées et forment une antithèse fondée sur la différence des objets, qui ne vient en pleine lumière que si l'on conserve le même verbe (art. cit., p. 154). J'ai dit plus haut que l'interprétation usuelle du passage dépend de la correction que je suis en train d'examiner. Mais il est clair que ce deuxième argument en faveur de la correction, bien loin de pouvoir fonder l'interprétation du passage, dépend en réalité entièrement de la façon dont on le comprend. Si l'on oppose, comme Vahlen, kai houtoi (1127b25) à hoi de (1127b26), c'est qu'on distingue deux sortes d'eirônes, dont seule la deuxième encourt un blâme, blâme dont on exempte par là même Socrate qu'on isole dans la première catégorie. Malheureusement, il est difficile de faire passer là où le veut Vahlen la frontière entre les deux catégories d'eirônes. Difficile en effet d'admettre que le de de la ligne 26 marque une antithèse avec la phrase précédente, puisque celle-ci ne comporte aucun men, et commence au contraire par malista de. Un de succédant à un autre de ne marque généralement pas une opposition, mais a la valeur d'une simple particule d'enchaînement: «et ...». C'est au contraire la phrase qui commence par malista de qui paraît, plus vraisemblablement, être en opposition avec khahriesteroi men (1127b23). Il ne s'agit donc pas de deux catégories d'eirônes, mais de deux traits qui les caractérisent: apparemment «plus gracieux», les eirônes se signalent surtout par le fait qu'ils refusent leur assentiment auxendoxa - c'est-à-dire, rappelons-le, à ce qui fait, selon la définition des Topiques, l'accord de tous ou au moins des plus sages: d'où le blâme d'Aristote à l'égard de cette attitude, blâme qui englobe tout à fait normalement Socrate, constamment ignorant du consensus et appuyant par conséquent ses raisonnements dialectiques sur des prémisses mauvaises aux yeux d'Aristote: bien loin de l'épargner, c'est précisément Socrate qu'atteint le blâme d'Aristote à l'égard des eirônes.

Cette interprétation de ta endoxa aparnountai, on le notera, s'accommoderait très bien de la correction de Vahlen: sous-entendre, encore une fois dans son sens aristotélicien normal, aparnoumenoi au lieu de lire prospoioumenoi donnerait un sens très cohérent avec ce qui précède :

mais surtout ils (scil. les eirônes) refusent leur assentiment aux idées admises, comme le faisait Socrate. Et ceux qui refusent leur assentiment sur les points de détail et les choses évidentes...

On a envie d'ajouter ici encore «comme le faisait Socrate»: on aurait ainsi de bout en bout une dénonciation sous le nom d'ironie de la pratique dialectique de Socrate. Ce qui empèche d'aller dans ce sens, c'est l'exemple proposé cette fois pour illustrer le comportement consistant à ta mikra kai phanera prospoieisthai (ou aparneisthai, si l'on suit Vahlen) - comportement dont nous ne savons toujours pas ce que c'est. Cet exemple, c'est le vêtement des Spartiates - exemple dont l'interprétation usuelle ne sait que faire: quel rapport entre «dissimuler» ou «nier» posséder les choses petites et évidentes et le vêtement des Spartiates? Il n'est pas interdit d'y voir une nouvelle allusion à Socrate et à sa simplicité vestimentaire [2].C'est en tout cas l'une des anecdotes rapportées à ce sujet par Alcibiade (Symp. 220b5-8) qui peut le mieux nous aider à interpréter le sens de l'expression utilisée par Aristote: lors de l'expédition de Potidée, où l'armée eut à supporter un hiver particulièrement froid, Socrate, alors que chacun s'emmitouflait comme il pouvait, restait vêtu aussi simplement que d'habitude, allant même pieds nus sur la glace; mais cela n'avait d'autre effet que de le faire regarder de travers par les soldats, qui comprenaient sa conduite comme du mépris à leur égard. Le jugement d'Aristote paraît comme repris de celui de ces soldats: ta mikra kai phanera prospoieisthai, écrit-il, «paraît quelquefois de la vantardise, comme le vêtement des Spartiates (EN 1127b28). Rappelant qu'au livre II Aristote disait que les vantards exagèrent dans le sens du «plus», et les «ironistes» dans le sens du «moins», je propose de lire ici une explicitation de cette expression: les «ironistes» exagèrent «ce qu'ils ont de petit et de visible», autrement dit la modestie, voire le dénuement, de leur apparence, jusqu'à se livrer parfois ainsi à une forme de vantardise: dans la prospoiêsis, les extrêmes finissent par se toucher, et l'eirôneia par revenir au même que l'alazoneia [3]. Insolence (hubris) aux yeux de l'Alcibiade de Platon, vantardise chez Aristote: on ne reconnaît dans ces perceptions de l'ironie socratique ni la définition de Quintilien, pour qui ironiser c'est parler par antiphrase, ni la volonté de modestie qu'une idéalisation postérieure attribuera à Socrate. D'Aristophane à Théophraste, en passant par les interlocuteurs de Socrate dans Platon et par Aristote, nous sommes au contraire confrontés à un consensus hostile à l'ironie, comprise comme refus, voire mépris, des idées reçues, des valeurs établies et des usages convenus.

Qu'on s'en tienne à l'analyse aristotélicienne de l'ironie pratiquée par Socrate, ou que l'on procède à un relevé, dans le corpus platonicien, des occurrences des mots eirôn, eirôneia ou eirôneuesthai appliqués à Socrate, on sera conduit à la même observation: ils expriment un reproche adressé à Socrate aussi bien pour sa manière de vivre (le manteau spartiate chez Aristote, les pieds nus sur la glace à Potidée ou l'insensibilité à la beauté d'Alcibiade dans le récit fait par ce dernier) que pour sa façon de discuter (refuser les idées admises, chez Aristote; éviter de répondre, chez Platon). Tirer au clair la nature de l'ironie socratique revient donc à savoir ce qu'il y a de commun à ces différents traits, qui permet, bien qu'ils appartiennent à des registres différents, de les subsumer sous le même terme.

C'est Aristote encore qui le rappelle, «Socrate interrogeait mais ne répondait pas, car il reconnaissait ne pas savoir (Réfutations sophistiques 34, 183b7-8): c'était là peut-être le trait le plus connu de la dialectique socratique. Notons tout de suite l'affinité entre 
cette obstination à ne pas répondre et le refus des idées admises prêté par Aristote aux «ironistes et nommément à Socrate: refuser les idées admises, en déclarant ignorer, par exemple, ce qu'est la vertu ou le beau, c'est ce qui permet à Socrate, dans les dialogues platoniciens, de retourner la situation et de se retrouver, face à Ménon ou à Hippias, en position de questionneur. Ni Ménon ni Hippias, certes, ne parlent d'ironie, mais c'est exactement le même comportement de la part de Socrate qui amène Thrasymaque à se plaindre de «l'ironie habituelle de Socrate, hê eiôthuia eirôneia Sôkratous (Rep. I, 337a3); la suite du passage identifie de façon plus explicite encore refuser de répondre et «ironiser» — qui n'a en l'occurrence rien à voir avec «se moquer»:

je le savais, dit Thrasymaque, et je leur avais annoncé, que tu refuserais de répondre (apokrinasthai men ouk ethelêsois) mais que tu «ironiserais» (eirôneusoio de) et ferais tout plutôt que de répondre, si l'on t'interrogeait sur un point (loc. cit., a5-7).

Ce qu'il faut comprendre, c'est en quoi le refus de répondre, de la part de Socrate, est pour Thrasymaque un motif de plainte. Il faut pour cela replacer l'intervention de Thrasymaque dans son contexte. La question de la nature de la justice a surgi, on le sait, au détour de la conversation initiale de Socrate avec Céphale; mais Céphale s'étant éclipsé à ce moment, c'est son fils Polémarque qui prend sa place. Citant Simonide, selon qui il est juste de rendre à chacun ce qu'on lui doit, il en conclut que la justice consiste à faire du bien à ses amis, du mal à ses ennemis (331e-332b). Socrate, qui depuis le début interroge, réfute cette définition de la justice et contraint Polémarque à reconnaître qu'il n'est jamais juste de faire du mal à quelqu'un (335e). C'est à ce moment, le moment où, la première définition de la justice s'étant révélée insuffisante, Socrate en demande une meilleure, que se situe l'intervention de Thrasymaque, appelant Socrate à ne pas se contenter d'interroger et d'examiner les opinions d'autrui, mais à répondre lui aussi, c'est-à-dire, en l'occurrence, à dire ce qu'est selon lui la justice. Au lieu d'obéir à cette injonction, Socrate implore l'indulgence de Thrasymaque pour son incapacité de traiter convenablement la question, et c'est cette prière que Thrasymaque interprète comme un refus de répondre dans lequel il reconnaît «l'ironie habituelle de Socrate».

Pour comprendre ce que Thrasymaque met ici sous le mot «ironie», mon hypothèse est qu'il faut être attentif à la façon dont se succèdent les interlocuteurs de Socrate. Le premier, on l'a vu, était Céphale, mais à la première mise en question de son opinion par Socrate, il a préféré abandonner la discussion. Mais Socrate ne l'a pas laissé partir sans faire de son fils son «héritier» dans la discussion $(331 \mathrm{~d})$. Or il apparaît bientôt que ce dont Polémarque a hérité, ce n'est pas de l'opinion de son père, puisqu'il n'hésite pas à la reformuler pour mieux la défendre: c'est plutôt de son rôle, celui de répondant. En demandant à Céphale de désigner avant de partir un héritier, Socrate indique qu'il n'acceptera de poursuivre avec un autre la discussion qu'à la condition de rester pour sa part dans le même rôle, celui de questionneur. Ainsi, Polémarque entrant dans le rôle de son père (même si c'est pour donner d'autres réponses), c'est en réalité la même discussion qui se poursuit avec lui. Quand Thrasymaque interpelle Socrate, cette discussion est arrivée à son terme, Polémarque s'étant reconnu réfuté. L'appel de Socrate à une nouvelle définition ne s'adresse pas nécessairement à lui, mais à n'importe lequel des auditeurs qui pense en connaître une. Il n'est plus question ici de désigner à Polémarque à son tour un «héritier»: Polémarque réfuté, Socrate se cherche en réalité un nouvel interlocuteur. D’où la réplique de Thrasymaque: pourquoi ne pas changer, non seulement de partenaire, mais aussi de rôle?

Thrasymaque, indique Socrate, attendait depuis longtemps l'occasion de «s'emparer de la discussion»: antilambanesthai tou logou(336b), expression qui désigne couramment la position du questionneur (cf. Rep. 337e3; Gorg. 506a2; Hipp. mai. 287a4). Mais il ne peut le faire qu'à ce moment, celui où, la discussion avec Polémarque ayant atteint son terme, la possibilité est ouverte d'une nouvelle discussion et à cette occasion d'une redistribution des rôles: invitant Socrate à répondre, pour une fois, au lieu d'interroger, Thrasymaque se propose comme questionneur.

Que peut bien avoir d' «ironique» l'obstination de Socrate à se maintenir dans le rôle de questionneur et à replacer quiconque veut l'interroger — ici Thrasymaque, ailleurs Ménon, Hippias ou Polos — dans la position de répondant? Certainement pas le fait que les prétextes invoqués (l'ignorance de Socrate de la définition recherchée, ou ici son incapacité d'examiner la question correctement) soient peut-être fallacieux: quand il crie à l' «ironie», Thrasymaque ne s'en prend pas un instant à l'insincérité qui serait celle de Socrate; la dissimulation dont ferait preuve Socrate n'est à tout prendre qu'un moyen en vue d'une fin, ne pas répondre, et c'est la poursuite de cette fin que Thrasymaque appelle «ironie».

Car Socrate ne se contente pas de refuser de répondre: dans une dialectique qui ne connaît que les deux rôles de questionneur et de répondant, ne pas vouloir ou dire, comme le fait Socrate, ne pas pouvoir répondre consacre normalement la victoire du questionneur. Ce qui est frappant chez Socrate, c'est qu'il parvient à éviter de répondre sans avoir pour autant à s'avouer battu: il tourne son refus de répondre dans la forme d'une question, renversant ainsi les rôles, imposant sa règle à la discussion au lieu de se soumettre à celle que tous acceptent et que son interlocuteur est par conséquent en droit de s'attendre à le voir respecter. L' ironie de Socrate, en résumé, n'a aux yeux de Thrasymaque rien à voir avec la question de sa sincérité: eirôneia est le nom qu'il donne à la façon qui est celle de Socrate de se dérober aux obligations que lui assignent les règles d'un entretien codifié, règles connues de tous et permettant pour cette raison de juger du bon déroulement de cette discussion. C'est pourquoi la meilleure traduction d'eirôneuesthai me paraît être, plutôt qu' «ironiser», «se dérober», et celle d'eirôneia, «dérobade». C'est d'ailleurs précisément dans ce sens, on le notera, que Démosthène emploie le mot eirôneia pour reprocher aux Athéniens de se dérober à leurs obligations fiscales ou militaires dans la lutte contre Philippe de Macédoine (Phil. I., 7).

À l'appui de cette analyse, j'invoquerai le passage du Gorgias auquel j'ai fait allusion plus haut, où Calliclès et Socrate se renvoient 
l'un à l'autre l'accusation d'eirôneuesthai(Gorg. 489e1 et 3) — il s'agit de l'un des rares cas où le mot figure dans la bouche de Socrate, lequel, à vrai dire, ne fait que retourner le reproche qui vient de lui être fait. L'occurrence du terme dans la bouche de Calliclès est d'ailleurs d'autant plus significative que le même Calliclès s'inquiète ailleurs (Gorg. 481b-c) de savoir si, oui ou non, Socrate plaisante, paizei, ce qui nous permet de faire la différence, dans la bouche du même locuteur, entre les deux verbes, «ironiser» et plaisanter ou se moquer.Eirôneuei, lance Calliclès à Socrate au moment où celui-ci, confronté à la thèse du droit du plus fort, opère un déplacement du plus fort au meilleur: au moment, donc, où Calliclès, qui a très clairement fixé l'objet de la discussion, le problêma, se voit lui aussi trompé dans son attente. À quoi Socrate répond qu'en matière d'ironie, il a été devancé, dans le moment où Calliclès, endossant le rôle de Zéthos dans l'Antiope d'Euripide, lui assignait le rôle d'Amphion (Gorg. 484 e, 485 e). Zéthos se moquait-il d'Amphion dans la pièce d'Euripide? Nous en savons trop peu pour en être sûrs. Quand il lui dit son envie de le gifler, Calliclès, lui, menace Socrate plus qu'il ne se moque de lui. Pour comprendre le diagnostic d'ironie porté après coup sur l'épisode par Socrate, on trouvera une indication plus sûre dans le fait que la référence à Euripide a servi à Calliclès à répartir des rôles entre Socrate et lui-même: l'ironie de Calliclès aura consisté à prévenir Socrate dans la distribution de leurs rôles respectifs (lui-même dans le rôle de Zéthos, l'apôtre de la vie active, face à Socrate dans le rôle d'Amphion, l'artiste ou le servant des Muses), tout comme l'ironie reprochée par Thrasymaque à Socrate consistait à ignorer la distribution des rôles dont Thrasymaque venait de prendre l'initiative. L'ironie de Calliclès, en d'autres termes, aura consisté à délimiter au départ de la discussion son rôle et celui de Socrate, en donnant pour cadre à la discussion une opposition toute faite, dont il emprunte le paradigme à la pièce d'Euripide, entre vie active et, sinon vie contemplative, du moins une vie consacrée au culte des Muses: culte que bien entendu ne dénierait pas Socrate, mais qu'il refuserait, qu'en face de Calliclès il va refuser, de voir tout bonnement opposer à l'action et à la politique. Car ce n'est pas seulement à réfuter le droit du plus fort que va s'employer Socrate, mais à prouver que c'est lui, le philosophe, qui est le vrai politique — au point de prétendre être, on le sait, le seul homme politique d'Athènes (Gorg. 521d) —, échappant ainsi au trop commode partage des rôles initialement institué par Calliclès.

Concluons. L'eirôneia n'a trait, en définitive, ni à la sincérité de l'intention, ni à la clarté des paroles et moins encore à leur véracité; elle n'a affaire ni avec la dissimulation, ni avec le double sens; l'eirôneia a trait à la tactique: c'est un stratagème qui se déploie en pleine lumière, aux yeux de tous, c'est-à-dire sans aucune dissimulation. Ce stratagème, on s'en rend compte par le dernier exemple cité, n'est pas le monopole de Socrate; Socrate en est cependant, au moins dans les dialogues de Platon, le principal utilisateur, et surtout il l'utilise d'une façon qui peut-être lui est propre, ce qui expliquerait que l'adjectif «socratique» fasse office d'épithète homérique ausitôt qu'on prononce le mot «ironie». Par le stratagème que j'ai décrit, en effet, Socrate parvient, à la surprise — et à la déception — générales, à se conduire à l'inverse de ce que les usages autorisent à attendre de lui. Qu'il s'agisse de répondre aux questions des sophistes ou aux avances d'Alcibiade, Socrate sait se dérober sans perdre la face; il réalise ce tour de force de se dérober sans quitter la partie: c'est probablement ce que désignait initialement le terme eirôn, quand Aristophane l'employait comme une sorte de marque de fabrique du socratisme, entre deux adjectifs désignant la souplesse du cuir et la viscosité de la peau huileuse d'un athlète, qui lui permet de glisser entre les mains de son adversaire (Nub. 449): masthlês, eirôn, gloios, à l'école de Socrate, tu deviendras «souple, prompt à l'esquive, glissant».

\section{MICHEL NARCY}

Centre National de la Recherche Scientifique, Villejuif.

France

Footnotes

[1] Gregory Vlastos, Socrates: Ironist and Moral Philosopher, Cambridge 1991 (trad. fr. par Catherine Dalimier, Socrate. Ironie et philosophie morale, Paris, 1994), chap. I.

[2] Outre l'anecdote rappelée ci-après, on peut en effet rappeler l'épigramme d'Ameipsias rapportée par Diogène Laërce II 28.

[3] D'où peut-être l'ellipse de l'eirôneia, complétée par Immelman, au début du chapitre (1127 a 13-4). Si l'eirôneia n'est en fin de compte qu'une forme d'alazoneia, Aristote peut parler de «la médiété de la vantardise». 